\title{
Assessing the Firing Properties of the Electrically Stimulated Auditory Nerve Using a Convolution Model
}

\author{
Stefan B. Strahl, Dyan Ramekers, Marjolijn M. B. Nagelkerke, \\ Konrad E. Schwarz, Philipp Spitzer, Sjaak F. L. Klis, Wilko Grolman \\ and Huib Versnel
}

\begin{abstract}
The electrically evoked compound action potential (eCAP) is a routinely performed measure of the auditory nerve in cochlear implant users. Using a convolution model of the eCAP, additional information about the neural firing properties can be obtained, which may provide relevant information about the health of the auditory nerve. In this study, guinea pigs with various degrees of nerve degeneration were used to directly relate firing properties to nerve histology. The same convolution model was applied on human eCAPs to examine similarities and ultimately to examine its clinical applicability. For most eCAPs, the estimated nerve firing probability was bimodal and could be parameterised by two Gaussian distributions with an average latency difference of $0.4 \mathrm{~ms}$. The ratio of the scaling factors of the late and early component increased with neural degeneration in the guinea pig. This ratio decreased with stimulation intensity in humans. The latency of the early component decreased with neural degeneration in the guinea pig. Indirectly, this was observed in humans as well, assuming that the cochlear base exhibits more neural degeneration than the apex. Differences between guinea pigs and humans were observed, among other parameters, in the width of the early component: very robust in guinea pig, and
\end{abstract}

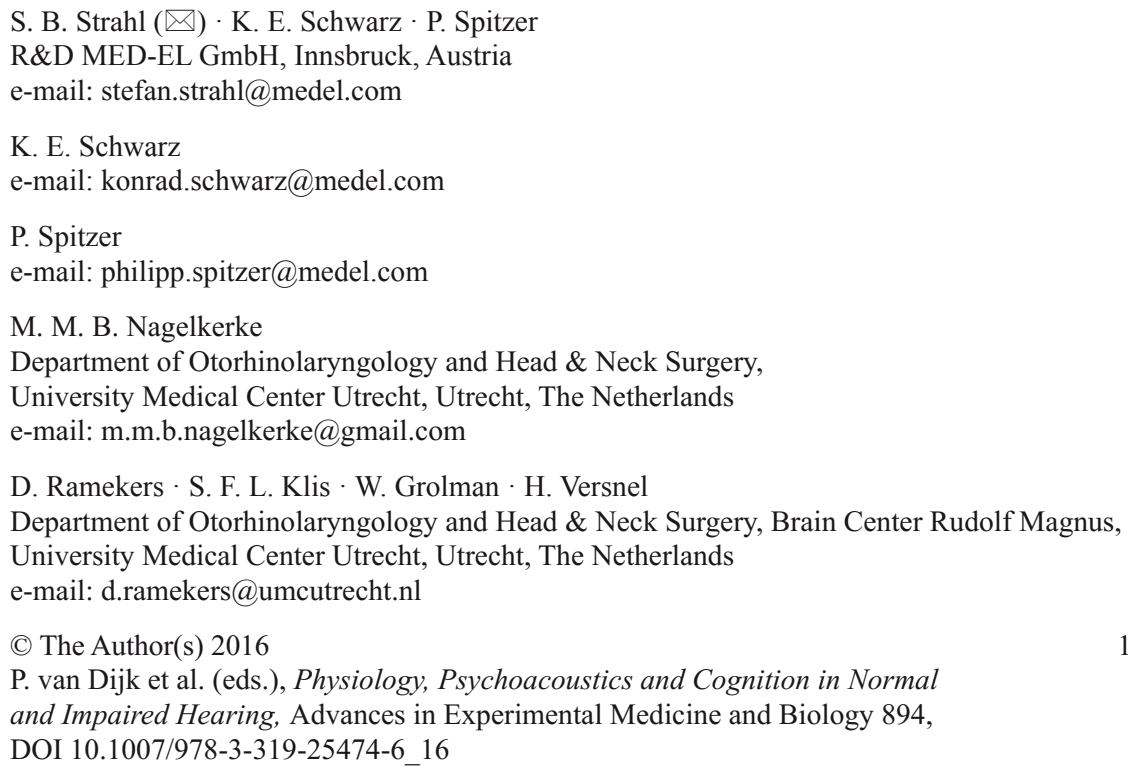


dependent on stimulation intensity and cochlear region in humans. We conclude that the deconvolution of the eCAP is a valuable addition to existing analyses, in particular as it reveals two separate firing components in the auditory nerve.

Keywords Auditory nerve $\cdot$ eCAP $\cdot$ Cochlear implant $\cdot$ Deconvolution $\cdot$ Firing probability $\cdot$ Neural health

\section{Introduction}

Most cochlear implant (CI) systems allow for the recording of the auditory nerve's response to an electric stimulus - the electrically evoked compound action potential (eCAP). This objective measure is an important clinical tool to assess the quality of the electrode-nerve interface of a CI recipient (Miller et al. 2008). Routinely, the lowest stimulation level that evokes an eCAP ('threshold') is determined. The morphology of suprathreshold eCAP waveforms is usually not evaluated.

\subsection{Mathematical Model of the Compound Action Potential}

The recorded compound action potential $(C A P)$ is described as the convolution of the unit response ( $U R$, i.e., the waveform resulting from a single action potential) with the compound discharge latency distribution $(C D L D)$, the sum of spike events over time of all individual nerve fibres (Goldstein and Kiang 1958, see also Fig. 1).

$$
\operatorname{CAP}(\mathrm{t})=\int_{-\infty}^{\mathrm{t}} \operatorname{CDLD}(\tau) \mathrm{UR}(\mathrm{t}-\tau) \mathrm{d} \tau
$$

This mathematical model was validated with simultaneous recordings of acoustically evoked CAPs from the round window and single-fibre responses from the auditory nerve in guinea pigs (Versnel et al. 1992b).

Most mathematical models of the CAP are concerned with solving the forward problem, i.e. predicting the CAP by modelling the activation of single nerve fibres and assuming a convolution with the unit response (Teas et al. 1962; de Boer 1975; Elberling 1976b; Kiang et al. 1976; Versnel et al. 1992b; Frijns et al. 1996; Miller et al. 1999; Briaire and Frijns 2005).

\footnotetext{
S F.L. Klis

e-mail: s.klis@umcutrecht.nl

W. Grolman

e-mail:w.grolman@umcutrecht.nl

H. Versnel

e-mail: h.versnel@umcutrecht.nl
} 

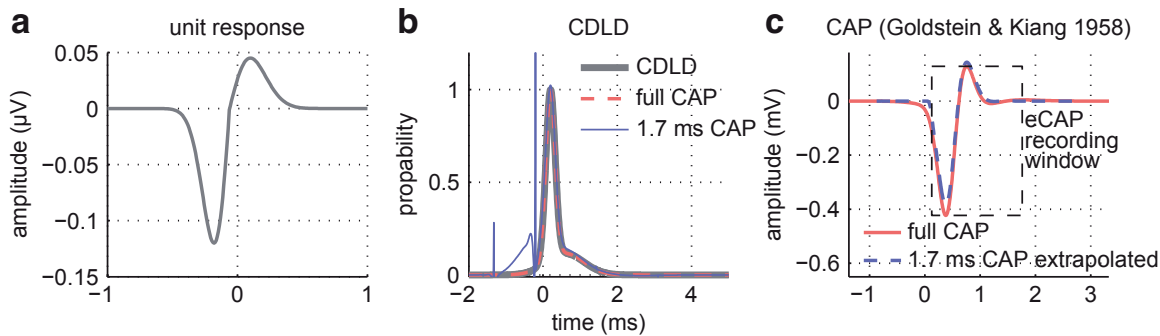

Fig. 1 The assumed elementary unit response of a single nerve fibre is shown in a. Its convolution with a CDLD (b, thick gray line) results in the CAP waveform shown in $\mathbf{c}$ (red line). The monotonic extrapolation of the time-limited eCAP recording is shown in $\mathbf{c}$ (blue dashed line). The derived CDLDs obtained by Eq. 4 are shown in $\mathbf{b}$ for the full eCAP (red dashed line) and a simulation of the time-limited and extrapolated eCAP (thin blue line)

A few studies addressed the inverse problem, i.e. predicting the firing probability by deconvolution of acoustically evoked CAPs with a unit response (Elberling 1976a; Charlet de Sauvage et al. 1987). To our knowledge no study investigated the inverse problem for eCAPs. In this study, we perform a deconvolution of eCAPs to examine CDLDs, presuming that they could provide information about the neural status of the auditory periphery. It has been shown that the eCAP in a guinea pig exhibits differences in its morphology depending on the neural health of the auditory nerve (Ramekers et al. 2014, 2015). Therefore, eCAPs from deafened guinea pigs are analysed to assess effects of the status of the auditory nerve on its firing probability. eCAPs from human CI users are evaluated for comparison to the effects observed in the animal model.

\section{Methods}

\section{1 eCAP Recording System}

eCAP measurements were performed with MED-EL PULSAR or SONATA CIs (for details see Neustetter et al. 2012). A charge-balanced biphasic pulse with an interphase gap of $2.1 \mu \mathrm{s}$ and a phase duration of $30 \mu \mathrm{s}$ was presented at an intra-cochlear stimulation electrode in a monopolar configuration. The stimulation amplitude was defined in current units (cu), where $1 \mathrm{cu}$ corresponds to approximately $1 \mu \mathrm{A}$. The eCAP was recorded on a neighbouring electrode with a minimum delay of $125 \mu \mathrm{s}$ from stimulus onset to reduce stimulation artefacts. The recording window was $1.7 \mathrm{~ms}$. Single eCAP recordings were separated by at least $20 \mathrm{~ms}$, assuming a relative refractory time below $10 \mathrm{~ms}$ (Brill et al. 2009; Ramekers et al. 2015). Any stimulation or recording artefact was removed using an alternating polarity and zero amplitude template paradigm, respectively (for details see Brill et al. 2009). 


\section{2 eCAP Data Sets}

\subsubsection{Guinea Pig}

Data were acquired from 18 guinea pigs (Ramekers et al. 2014, 2015), which were divided into 3 groups of 6 animals: normal-hearing (NH), 2 weeks deaf (2WD) and 6 weeks deaf (6WD). Methods of deafening and eCAP recordings are described in detail in Ramekers et al. (2014).

Briefly, profound cochlear hair cell loss was induced by co-treatment of kanamycin and furosemide. It resulted in mild degeneration of the auditory nerve after 2 weeks ( $25 \%$ loss of spiral ganglion cells, SGCs) and severe degeneration after 6 weeks ( $60 \%$ loss of SGCs).

The eCAP recordings were performed in isoflurane-anaesthetized animals. eCAPs were evoked and recorded with two electrodes placed in the basal turn through a cochleostomy. In this study, three different stimulation intensities were analysed: just above threshold (first visible eCAP), halfway the input/output function ('intermediate'), and at maximum stimulation intensity typically corresponding to saturation $(800 \mathrm{cu})$. Waveforms were averaged over 900 iterations.

\subsubsection{Human}

From a multicentre study (Senn et al. 2012) eCAPs recorded post-operatively with the clinical system software MAESTRO (MED-EL GmbH, Innsbruck) in 52 awake human subjects were selected. The selection criteria were the availability of a preoperative audiogram and eCAPs of at least $100 \mu \mathrm{V}$ amplitude. The mean age at onset of hearing loss was 19 years (range: $0-72$ ). The mean age at implantation was 46 years (range: $15-79$ ).

The recordings were manually analysed by three experts. Similar to the animal model, three different stimulation levels were selected: threshold, intermediate, and maximum. In contrast to the animal model, maximum typically corresponded to the loudest acceptable presentation level. Intermediate corresponded to $50 \%$ eCAP amplitude compared to maximum.

The eCAPs were obtained from stimulation electrodes in the apical (contact 2), middle (contact 4 or 5) and basal cochlear region (contact 9 or 10). Waveforms were averaged over 25 iterations and $5 \mathrm{kHz}$ low-pass filtered (fifth-order Butterworth) to remove any remaining recording noise.

\subsection{Deconvolution of the eCAP}

Following Eq. (1) and Versnel et al. (1992a) the unit response UR is assumed constant and was modelled by Eqs. 2 and 3 with $U_{N}=0.12 \mu \mathrm{V}, \sigma_{\mathrm{N}}=0.12 \mathrm{~ms}$ describing 
the negative and $U_{P}=0.045 \mu \mathrm{V}, \sigma_{P}=0.16 \mathrm{~ms}$ describing the positive part. The cross point is defined with $t_{0}=-0.06 \mathrm{~ms}$ (Fig. 1a).

$$
\begin{aligned}
& U R(t)=\frac{U_{N}}{\sigma_{N}}\left(t-t_{0}\right) e^{\frac{1}{2}-\frac{\left(t-t_{0}\right)^{2}}{2 \sigma_{N}^{2}}}, t<t_{0} \\
& U R(t)=\frac{U_{P}}{\sigma_{P}}\left(t-t_{0}\right) e^{\frac{1}{2}-\frac{\left(t-t_{0}\right)^{2}}{2 \sigma_{P}^{2}}}, t \geq t_{0}
\end{aligned}
$$

Having assumed the same unit response for all contributing fibres, the CDLD can be obtained directly from the recorded eCAP by deconvolution. The deconvolution was performed in the frequency domain with

$$
C D L D(t)=\mathcal{F}^{-1}\left(\frac{\mathcal{F}(e C A P(t))}{\mathcal{F}(U R(t))}\right)
$$

where $\mathcal{F}$ represents the Fourier transform and $\mathcal{F}^{-1}$ the inverse Fourier transform.

The baseline was estimated over the final $200 \mu$ s and, assuming that the eCAP decays monotonically to baseline before and after the recording window, the eCAPs were linearly extrapolated to baseline (Fig. 1c) before performing the deconvolution. The extrapolation only affected the CDLD before stimulus onset (see Fig. 1b). The deconvolution of the eCAP can be understood as applying an inverse lowpass filter, amplifying unwanted high-frequency noise. Therefore, the extrapolated eCAPs were low-pass filtered by a 50-point moving-average filter applied twice (once in forward and once in reverse direction) to achieve a zero-phase digital filtering. Any remaining high-frequency noise in the CDLD was removed by a $2.5 \mathrm{kHz}$ fifth-order Butterworth low-pass filter.

\subsection{Parametrisation of the CDLD}

The CDLDs derived from eCAP responses from both data sets exhibited a skewed, quasi-bimodal, distribution (see Fig. 2). To perform further analysis, a parametrisation of the CDLD was performed by a two-component Gaussian mixture model (GMM).

$$
C D L D=a_{1} \mathcal{N}\left(\mu_{1}, \sigma_{1}\right)+\mathrm{a}_{2} \mathcal{N}\left(\mu_{2}, \sigma_{2}\right)
$$

The GMM was fitted to the CDLDs using a nonlinear least-squares regression (nlinfit, MATLAB; Mathworks, Natick, MA, USA) with initial start values being manually optimised if needed to achieve an adequate fit $\left(\mathrm{R}^{2}>0.95\right)$. As outcome parameters we considered $\mu_{1}$ and $\mu_{2}$ (corresponding to the peak latencies), $\sigma_{1}$ and $\sigma_{2}$ (reflecting peak width), and the ratio of the components $a_{2} / a_{1}$. 


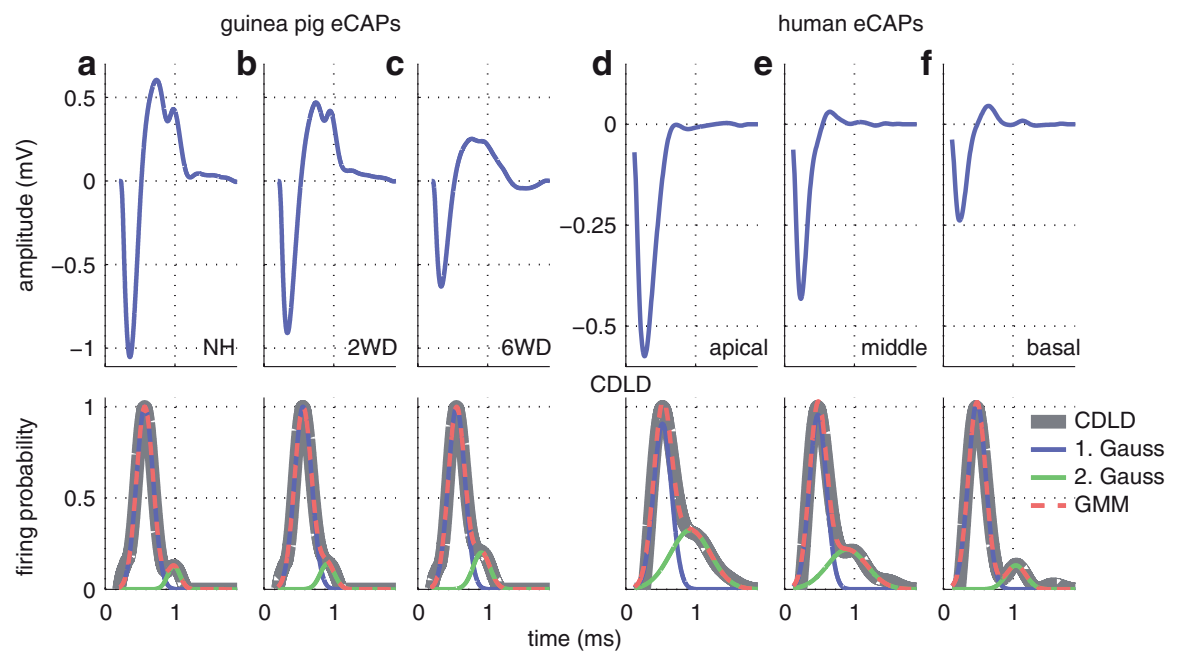

Fig. 2 Example eCAPs for maximum stimulation level (upper row) and the corresponding CDLDs and GMM fits (bottom row). The left three panels show examples of each of the three guinea pig groups; the right three panels are from each of the three cochlear regions in one human subject

\section{Results}

\subsection{Guinea Pig Deafness Model eCAPs}

Figure 2 shows eCAP and CDLD examples for NH (a), 2WD (b) and 6WD animals (c). In each animal the deconvolution of the recorded eCAPs resulted in CDLDs with a fast rising peak, typically narrow $(<0.5 \mathrm{~ms}$ width), in most cases followed by a shoulder-shaped component or a second peak. In those cases the CDLD could be well fitted with the two-component GMM (Eq. 5), remaining single-peak cases ( $8 \%$ of all CDLDs) could be well fitted with a single Gaussian. Figure 3 and Table 1 show group averages of CDLD parameters. Two variables significantly varied with group (i.e., with degeneration of the auditory nerve): $\mu_{1}$ and $\mathrm{a}_{2} / \mathrm{a}_{1}(p<0.05$; rmANOVA with stimulation intensity as within factor, and group as between factor). With more nerve degeneration (6WD vs. $2 \mathrm{WD}$ and $\mathrm{NH}$ ) the peak latency $\mu_{1}$ was shorter and $\mathrm{a}_{2} / \mathrm{a}_{1}$ was larger.

Table 1 CDLD parameters for guinea pig groups (upper section), and humans grouped by cochlear region (lower section), averaged over stimulation level

\begin{tabular}{l|l|l|l|l|l|l}
\hline \multicolumn{2}{c}{} & $\mu_{1}(\mathrm{~ms})$ & $\mu_{2}-\mu_{1}(\mathrm{~ms})$ & $\sigma_{1}(\mathrm{~ms})$ & $\sigma_{2}(\mathrm{~ms})$ & $\mathrm{a}_{2} / \mathrm{a}_{1}$ \\
\hline \multirow{4}{*}{ Guinea pig } & $N H$ & 0.58 & 0.38 & 0.14 & 0.10 & 0.090 \\
\cline { 2 - 7 } & $2 W D$ & 0.60 & 0.36 & 0.16 & 0.10 & 0.068 \\
\cline { 2 - 7 } & $6 W D$ & 0.52 & 0.37 & 0.14 & 0.13 & 0.33 \\
\hline \multirow{4}{*}{ Human } & apex & 0.54 & 0.42 & 0.13 & 0.27 & 0.96 \\
\cline { 2 - 7 } & middle & 0.50 & 0.42 & 0.14 & 0.27 & 0.86 \\
\cline { 2 - 7 } & base & 0.51 & 0.46 & 0.14 & 0.26 & 0.85 \\
\hline
\end{tabular}



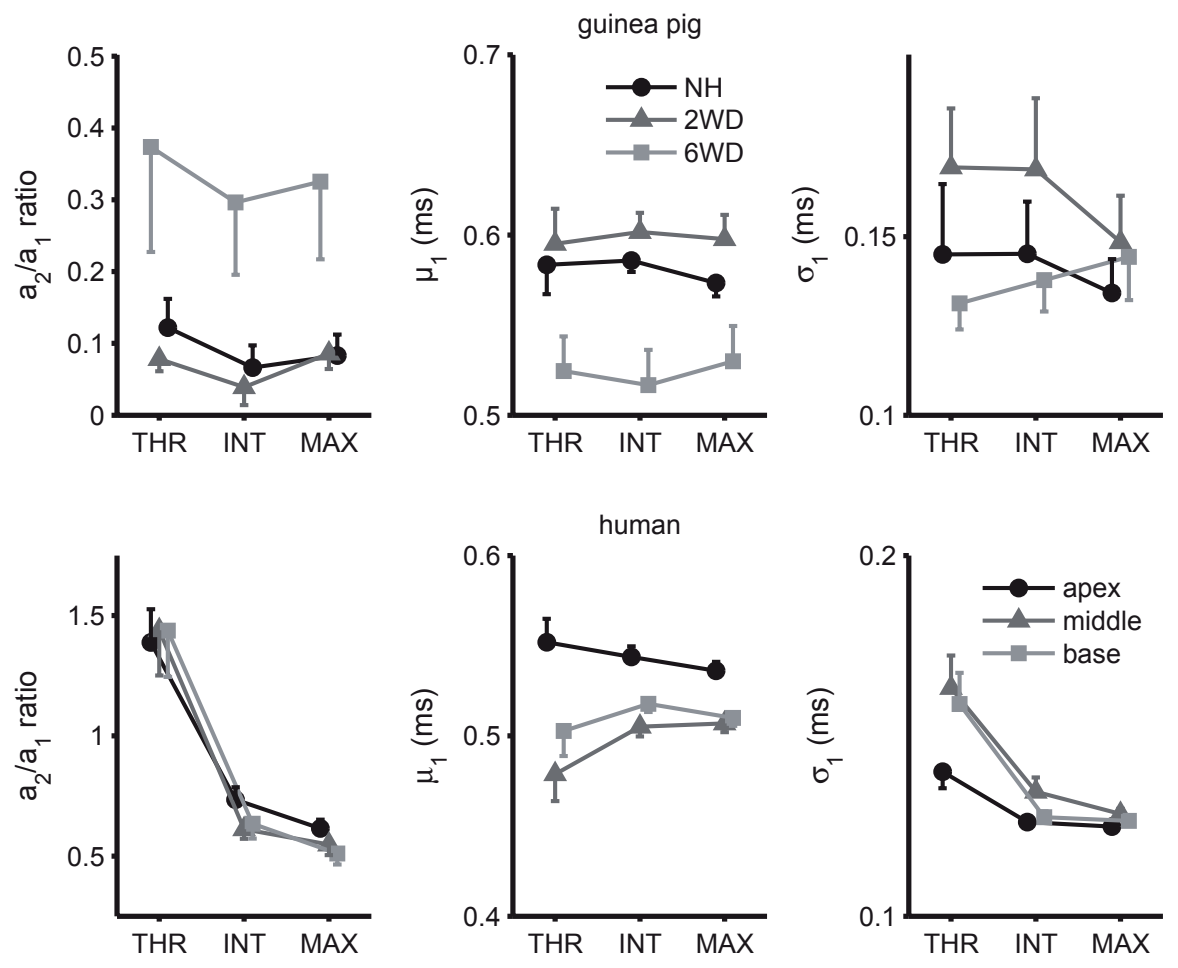

\section{stimulation intensity}

Fig. 3 Average CDLD parameters $\mathrm{a}_{2} / \mathrm{a}_{1}, \mu_{1}$ and $\sigma_{1}$ shown for guinea pigs (left) and humans (right). THR threshold, INT intermediate, MAX maximum. Error bars are SEM

\subsection{Human Clinical eCAPs}

Figure 2 shows examples of eCAPs and corresponding CDLDs for an apical (d), middle (e) and basal (f) stimulation site in one human subject. Human CDLDs showed a morphology comparable to the guinea pig data, with $15 \%$ of CDLDs being single-peaked. Figure 3 and Table 1 show averages of CDLD parameters for different cochlear regions. Assuming that any residual hearing correlates with the auditory nerve's neural health, subjects were divided into two groups having pre-operative $0.5-4 \mathrm{kHz}$ pure tone averages $\leq 105 \mathrm{~dB}$ hearing loss (HL) (24 subjects) or $>105 \mathrm{~dB}$ HL (28 subjects). No significant between-subject group effects were found ( $p=0.054$; rmANOVA with cochlear region and stimulation intensity as within factors, and group as between factor). A significant decrease of $a_{2} / a_{1}$ with increasing stimulation intensity $(p<0.001)$ and distance from the apex (apex vs. middle and base, $p<0.05$ ) was found. The latency $\mu_{1}$ was significantly longer in the 
apical cochlear region compared to middle and base $(p<0.001)$. The width of the first Gaussian $\sigma_{1}$ was significantly smaller in the apex vs. middle and base $(p<0.05)$ and with increasing stimulation intensity $(p<0.001)$.

\section{Discussion}

\subsection{Comparing Deconvolution Results from Guinea Pigs and Humans}

We observed several similarities between the obtained CDLDs of guinea pigs and humans. First, the CDLDs could be described in the large majority of cases by two Gaussian distributions (Fig. 2). Second, the peak latency of the early component decreased with increasing neural degeneration, assuming for the human data more degeneration at the cochlear base than near the apex (Fig. 3, $\mu_{1}$ ). Notable differences were observed in particular with respect to the width of the early component, which was very robust in guinea pigs, and quite variable with stimulation intensity and cochlear region in humans.

\subsection{Choice of UR Waveform}

The main challenge of the eCAP deconvolution is the dependence of the CDLDs on the choice of UR. For instance, with a larger $\mathrm{U}_{\mathrm{P}} \mathrm{a}_{2} / \mathrm{a}_{1}$ increases since the $\mathrm{P}_{2}$ component of eCAP can be reduced by increasing the $\mathrm{N}_{2}$. We have chosen our parameters based on experimental estimates of the UR in guinea pigs (Prijs 1986; Versnel et al. 1992a). Therefore, we can be fairly confident about our choice for the eCAP deconvolutions in the guinea pig. The UR in humans is thought to be similar (Briaire and Frijns 2005; Whiten 2007). However, the cochlear morphology is quite different in humans. The UR may change with stimulation site in the human cochlea and explain in part the cochlear region dependent variation of the CDLD seen in the human dataset. Also our assumption of the same UR across the nerve population might be too coarse (Westen et al. 2011), in particular considering location-dependent effects of degeneration, such as demyelination and cell shrinkage. To further address this point, a sensitivity analysis of the convolution model is needed.

\subsection{Parametrisation of the CDLD}

Single-fibre responses to electric pulses (estimated by post-stimulus time histograms) showing a fast rise and a slow decline (van den Honert and Stypulkowski 1984; Sly et al. 2007) may be modelled best with a gamma function (Versnel 
et al. 1992b). However, the distribution of response latencies between fibres may be Gaussian. Therefore, we tested fits of CDLDs with gamma functions and with Gaussian functions, and both in guinea pigs and humans we observed superior fits with Gaussian functions. Due to the large population size and assuming that the discharge jitter is smaller within than between fibres, the resulting convolution of the gamma function with the Gaussian distribution could explain the Gaussian-like CDLD.

\subsection{Interpretation of CDLD Parameters}

In most cases $a_{2} / a_{1}$ is significant, in both guinea pigs and humans. This suggests either two subpopulations of fibres and/or neural elements (peripheral vs. central site of excitation) with different latencies and proportions, or repeated firings of the same neurons. The latter option is supported by the following arguments (see also in Ramekers et al. 2015). First, the interval between the two components, $\mu_{2}-$ $\mu_{1}$, is about $0.5 \mathrm{~ms}$, which is around the absolute refractory period estimated from masker-probe responses. Second, the $\mathrm{N}_{2}-\mathrm{N}_{1}$ interval of the eCAP waveform has been found to correlate with recovery measures (Ramekers et al. 2015).

Alternatively, a reduced relative contribution of the second component with increasing stimulation intensity and more basal position within the cochlea as observed in humans could be explained by the former option. The late component can be ascribed to excitation peripheral to the cell body (Stypulkowski and van den Honert 1984) which is thought to occur to a larger extent near threshold (Briaire and Frijns 2005).

\section{Conclusion}

Obviously, there are multiple differences in experimental settings and biology between the guinea pig and human, which may contribute to different CDLD outcomes. Among others, cause of deafness, cochlear anatomy, duration of deafness, and rate of neural degeneration differ, and there is awake vs. anaesthetized state, chronic vs. acute cochlear implantation, and long vs. short electrode array. Taking these differences into account, a few characteristics appeared to be quite robust across species and experimental setting. Importantly, the deconvolution of the eCAP revealed two separate firing components, which could not easily be detected from the eCAP waveforms themselves. The ratio of the components and the latency of the early component came out as potential markers to assess the condition of the auditory nerve. 
Open Access This chapter is distributed under the terms of the Creative Commons AttributionNoncommercial 2.5 License (http://creativecommons.org/licenses/by-nc/2.5/) which permits any noncommercial use, distribution, and reproduction in any medium, provided the original author(s) and source are credited.

The images or other third party material in this chapter are included in the work's Creative Commons license, unless indicated otherwise in the credit line; if such material is not included in the work's Creative Commons license and the respective action is not permitted by statutory regulation, users will need to obtain permission from the license holder to duplicate, adapt or reproduce the material.

\section{References}

Briaire JJ, Frijns JHM (2005) Unraveling the electrically evoked compound action potential. Hear Res 205(1-2):143-156

Brill S, Müller J, Hagen R, Möltner A, Brockmeier S-J, Stark T et al (2009). Site of cochlear stimulation and its effect on electrically evoked compound action potentials using the MED-EL standard electrode array. BioMed Eng OnLine 8(1):40

Charlet de Sauvage R, Aran JM, Erre JP (1987) Mathematical analysis of VIIIth nerve cap with a linearly-fitted experimental unit response. Hear Res 29(2-3):105-115

De Boer E (1975) Synthetic whole-nerve action potentials for the cat. J Acoust Soc Am 58(5):1030 1045

Elberling C (1976a) Deconvolution of action potentials recorded from the ear canal in man. In: Stevens SDG (ed) Disorders of auditory function II. Academic, London

Elberling C (1976b) Simulation of cochlear action potentials recorded from the ear canal in man. In: Rubin R, Elberling C, Salomon G (eds) Electrocochleography. University Park Press, Baltimore, pp 151-168

Frijns JHM, Schoonhoven R, Grote JJ (1996) The influence of stimulus intensity on spike timing and the compound action potential in the electrically stimulated cochlea: a model study. Proceedings of the 18th Annual International Conference of the IEEE engineering in medicine and biology society, 1996. Bridging Disciplines for Biomedicine, vol 1, pp 327-328

Goldstein JMH, Kiang NY-S (1958) Synchrony of neural activity in electric responses evoked by transient acoustic stimuli. J Acoust Soc Am 30(2):107-114

Kiang NYS, Moxon EC, Kahn AR (1976) The relationship of gross potentials recorded from the cochlea to single unit activity in the auditory nerve. In: Ruben RJ, Elberling C, Salomon G (eds) Electrocochleography. University Park Press, Baltimore, pp 95-115

Miller CA, Abbas PJ, Rubinstein JT (1999) An empirically based model of the electrically evoked compound action potential. Hear Res 135(1-2):1-18

Miller CA, Brown CJ, Abbas PJ, Chi S-L (2008) The clinical application of potentials evoked from the peripheral auditory system. Hear Res 242(1-2):184-197

Neustetter C, Zangerl M, Spitzer P, Zierhofer C (2012) In-vitro characterization of a cochlear implant system for recording of evoked compound action potentials. BioMed Eng OnLine 11(1):22

Prijs VF (1986) Single-unit response at the round window of the guinea pig. Hear Res 21(2):127133

Ramekers D, Versnel H, Strahl SB, Klis SFL, Grolman W (2015) Recovery characteristics of the electrically stimulated auditory nerve in deafened guinea pigs: relation to neuronal status. Hear Res 321:12-24

Ramekers D, Versnel H, Strahl SB, Smeets EM, Klis SFL, Grolman W (2014) Auditory-nerve responses to varied inter-phase gap and phase duration of the electric pulse stimulus as predictors for neuronal degeneration. J Assoc Res Otolaryngol 15(2):187-202

Senn P, van de Heyning PL, Arauz S, Atlas M, Baumgartner W-D, Caversaccio M et al (2012) Electrically evoked compound action potentials in patients supplied with CI. Presented at the 
12th International Conference on cochlear implants and other implantable auditory technologies, Baltimore

Sly DJ, Heffer LF, White MW, Shepherd RK, Birch MGJ, Minter RL et al (2007) Deafness alters auditory nerve fibre responses to cochlear implant stimulation. Eur J Neurosci 26(2):510-522

Stypulkowski PH, van den Honert C (1984) Physiological properties of the electrically stimulated auditory nerve. I. Compound action potential recordings. Hear Res 14(3):205-223

Teas DC, Eldridge DH, Davis H (1962) Cochlear responses to acoustic transients: an interpretation of whole-nerve action potentials. J Acoust Soc Am 34(9, Pt. II):1438-1459

van den Honert C, Stypulkowski PH (1984) Physiological properties of the electrically stimulated auditory nerve. II. Single fiber recordings. Hear Res 14(3):225-243

Versnel H, Prijs VF, Schoonhoven R (1992a) Round-window recorded potential of single-fibre discharge (unit response) in normal and noise-damaged cochleas. Hear Res 59(2):157-170

Versnel H, Schoonhoven R, Prijs VF (1992b) Single-fibre and whole-nerve responses to clicks as a function of sound intensity in the guinea pig. Hear Res 59(2):138-156

Westen AA, Dekker DMT, Briaire JJ, Frijns JHM (2011) Stimulus level effects on neural excitation and eCAP amplitude. Hear Res 280(1-2):166-176

Whiten DM (2007) Electro-anatomical models of the cochlear implant (Thesis). Massachusetts Institute of Technology. http://dspace.mit.edu/handle/1721.1/38518. Accessed 1 April 2015 